\title{
Evolution and mechanisms of long life and high fertility in queen honey bees
}

\author{
Silvia C. Remolina • Kimberly A. Hughes
}

Received: 22 April 2008 / Accepted: 6 May 2008 /Published online: 22 June 2008

(C) American Aging Association 2008

\begin{abstract}
Honey bees (Apis mellifera) are eusocial insects that exhibit striking caste-specific differences in longevity. Queen honey bees live on average 1-2 years whereas workers live on average 15-38 days in the summer and 150-200 days in the winter. Previous studies of senescence in the honey bee have focused on establishing the importance of extrinsic mortality factors (predation, weather) and behavior (nursing and foraging) in worker bee longevity. However, few studies have tried to elucidate the mechanisms that allow queen honey bees to achieve their long lifespan without sacrificing fecundity. Here, we review both types of studies and emphasize the importance of understanding both proximate and ultimate causes of the unusual life history of honey bee queens.
\end{abstract}

Keywords Eusociality · Evolution · Fecundity . Honey bee $\cdot$ Lifespan $\cdot$ Senescence

\section{Abbreviations \\ IIS Insulin insulin-like signaling pathway \\ JH Juvenile hormone \\ ROS Reactive oxygen species \\ $\mathrm{Vg}$ Vitellogenin}

S. C. Remolina $(\square) \cdot$ K. A. Hughes

Department of Animal Biology and Institute for Genomic

Biology, University of Illinois at Urbana-Champaign,

1206 W. Gregory,

Urbana, IL 61801, USA

e-mail: remolina@uiuc.edu

\section{Introduction}

Advanced insect societies, such as those of ants, bees, wasps, and termites, are characterized by overlapping generations of adult colony members, a system of caste division (reproductive vs non-reproductive individuals), and cooperative care for young colony members (Wilson 1971). In these insects, queens and workers exhibit up to a 100-fold difference in lifespan, with reproductive queens outliving non-reproductive workers (Winston 1987; Keller and Genoud 1997; Page and Peng 2001). Social insect species are promising subjects for the study of aging, in part because of their caste-specific life-histories and extreme lifespan differences, which provide the opportunity to identify naturally occurring candidate genes involved in aging (Keller and Jemielity 2006).

Even more compelling is the observation that social insect queens live at least an order of magnitude longer than reproductive adults of non-social insects, despite sustaining high rates of reproduction. Longevity and fecundity typically are negatively correlated and the extent of this tradeoff varies within and among species. Social insect queens are the only animals known that can live for decades while also producing hundreds to thousands of offspring per day (Keller and Genoud 1997).

These observations raise several interesting questions. In this review, we will concentrate on the two we consider most fundamental. First, what evolutionary 
forces have produced these long-lived but highly fertile organisms? Second, what molecular mechanisms allow individuals to have extreme longevity and fecundity? We preface these discussions with a brief overview of honey bee (Apis mellifera) life history and caste-specific differences in lifespan and reproductive behavior.

\section{Life history, caste-specific lifespan and reproductive behavior}

Honey bees are social insects with a haplodiploid sex determination system, in which unfertilized eggs laid by the queen develop into drones (male bees) and fertilized eggs develop into either workers or queens (both castes consisting of genetically identical female bees). Queen-worker differentiation is nutritionally determined during the larval stage. Queens and drones are both reproductive, whereas workers are nonreproductive and spend their lives performing in-hive tasks or foraging outside the hive for pollen, nectar, and water.

All castes exhibit different developmental times and lifespans. Drones have a developmental time of 24 days, and they live on average 21-32 days in the spring and summer, the only times when drones are produced (Winston 1987). Workers develop in 21 days and their lifespans vary depending on season. Workers live on average 15-38 days in the summer (Free and Spencer-Booth 1959; Fukuda and Sekiguchi 1966), 30-60 days in the spring and fall, and 150-200 days in the winter (Anderson 1931; Free and SpencerBooth 1959; Fukuda and Sekiguchi 1966). Queens take the shortest time to develop (16 days) and have the longest lifespan. Queens live on average 1-2 years (Page and Peng 2001), although a maximum lifespan of 8 years was reported in one study (Bozina 1961). The dimorphism observed in the honey bee female caste is particularly interesting because workers and queens have the same genotype yet exhibit a 10 -fold difference in lifespan.

Queens become sexually mature 6 days after emergence and then take orientation flights and two or three mating flights per day. On average, they mate with 17 drones and store all of the sperm needed to fertilize eggs for the duration of their lifespan (Woyke 1960). After queens complete their mating flights, they return to the hive, where they engage in egg-laying activities
(Winston 1987). With the exception of the mating flights queens take early in life, they do not leave the protected hive environment except during colony fission. Queens can lay an impressive 1,500-2,000 eggs per day throughout their lives (Merrill 1924; Nolan 1925).

Although all worker honey bees are non-reproductive, their life histories are highly plastic and responsive to seasonality and the social and demographic composition of the hive (Robinson 1992). In temperate regions, worker life history depends strongly on season. In the warm season, workers spend the first 2 weeks of life in the hive performing tasks such as comb-building, cell-cleaning, and brood care ("nursing"), before transitioning to the foraging stage in which they fly distances of up to $21 \mathrm{~km}$ per day to collect nectar and pollen for the hive (Neukirch 1982). The nurse-forager transition is responsive to changing social conditions and can be accelerated, delayed, or even reversed depending on the demographic composition of the hive (Robinson 1992; Huang and Robinson 1996).

Caste differentiation for larvae that become queens or workers has been associated with the quantity and quality of food that larvae receive before the third larval instar. Larvae fed a rich diet composed of royal jelly develop into queens, whereas larvae fed a diet composed of glandular secretions, honey, and pollen develop into workers (Winston 1987). Physiologically, queen-destined larvae have high levels of juvenile hormone $(\mathrm{JH})$, which prevents ovarian apoptosis in 5th-instar queen larvae. JH levels have also been implicated in upregulating ecdysteroid titers, which, in turn, activate transcription of genes that direct differentiation to the queen pathway (Page and Peng 2001, and references therein). The insulin signaling and TOR (target of rapamycin) pathways act as central regulators in queen-worker differentiation (Wheeler et al. 2006; Patel et al. 2007).

The nurse-forager transition appears to be orchestrated by $\mathrm{JH}$, which exhibits an age-related increase in worker bees: nurses have low levels of $\mathrm{JH}$, and foragers have high levels of JH (Robinson et al. 1991). Nurses also possess well-developed hypopharyngeal glands used in feeding other colony members (Winston 1987) and have high levels of abdominal lipids (Toth and Robinson 2005), and vitellogenin (Rutz and Lüscher 1974; Corona et al. 2007). In contrast, bees emerging in the cold season ("winter" or diutinus 
bees) have a different physiological profile than bees emerging in the summer. Winter bees possess well developed hypopharyngeal glands, high vitellogenin levels, and low levels of JH (Fluri et al. 1977).

\section{Evolutionary and ecological determinants of lifespan}

Social insect queens, including queen honey bees, are extraordinarily long-lived compared both to nonsocial insects and workers of their own species. The combination of advanced sociality, long life and high fecundity is a syndrome that has evolved independently multiple times in insects, including termites, ants, and several species of bees. Although difficult to test experimentally, a plausible theory for the evolution of this syndrome has been proposed (Keller and Genoud 1997). A well-developed population-genetic model of the evolution of aging suggests that most multicellular organisms senesce because, while natural selection strongly opposes alleles that have detrimental effects on early life survival and fecundity, selection is very weak against alleles with equivalent effects that are confined to late life. Under this general model, either purely detrimental mutations or pleiotropic mutations with both beneficial and detrimental effects can contribute to the evolution of senescence, if the detrimental effects are confined to late ages. These two versions of the model are referred to as the Mutation Accumulation and Antagonistic Pleiotropy models, respectively (see Hughes and Reynolds 2005 and references therein for more detailed discussion of these models).

Under either version of the general evolutionary model, two demographic patterns can compensate for the decline in selection with age and hence promote long lifespan: low levels of extrinsic mortality in adult individuals and fecundity rates that increase with age (Charlesworth 1994; Baudisch 2005). Social insect colonies meet both these criteria. Adult queens are protected from predators and environmental extremes by the physical structure of their nests and defensive behaviors of non-reproductive workers. In addition, the reproductive output of social insect queens can increase dramatically with colony age (Hölldobler and Wilson 1990).

Another critical feature demonstrating the value of social insects as models for aging research is that the trade-off between longevity and reproductive output characterizing other taxa appears to be absent in social insects. In non-eusocial species of animals, these two traits are typically negatively correlated. Indeed, the existence of such tradeoffs is a cornerstone of life-history theory (Roff 2002; Stearns 1992). However, consideration of the ecology of social insects suggests a solution to this puzzle. Life-history tradeoffs are thought to arise from the need to partition limited resources among the competing demands of growth, survival, and reproduction. Because of their colonial life-style and division of labor, eusocial insects societies are thought to be able to garner more energy for the queen than a female of a non-social species would be able to collect (Wilson 1971; Keller 1998). If so, then life-history tradeoffs probably do occur in these species, but they are not apparent in contrast to other taxa because their resource budgets are much higher.

There have been few challenges to the general evolutionary model of social insect longevity (although the relative contributions of Mutation Accumulation and Antagonistic Pleiotropy processes have been debated, see Hughes and Reynolds 2005 and references therein). However, explanations for lifespan differences between queen and worker honey bees have received limited attention. One plausible hypothesis is that workers, once they become foragers and leave the protected hive environment, experience higher extrinsic mortality due to predation and accident (Neukirch 1982; Schmid-Hempel and Wolf 1988; Visscher and Dukas 1997). An alternate hypothesis is that queens and workers exhibit large differences in intrinsic patterns of aging. This is the view that motivates the use of comparisons between queens and workers as a tool for understanding the basic biology of aging (Parker et al. 2004; Corona et al. 2005, 2007). It is surprising, therefore, that there was little direct evidence to support differences in intrinsic patterns of aging until recently.

Early studies of honey bee aging established that worker bees emerging in the spring or fall lived longer than those emerging in the summer (Free and Spencer-Booth 1959; Fukuda and Sekiguchi 1966). A few studies pointed to intrinsic senescence as a cause of the worker-queen lifespan difference, but others implicated extrinsic mortality. Ribbands (1952) reported that bees that begin to forage later in life have shorter foraging careers and attributed 
this pattern to intrinsic senescence. Measurements of glycogen levels in free-living bees led Neukirch (1982) to propose that older foraging workers undergo a physiological change that directly affects life expectancy. Neukirch suggested that young foragers can replenish glycogen reserves, but that this ability is impaired in older foragers that exhaust their energy supplies and die in the field. Using experimental manipulation of foraging opportunity, Schmid-Hempel and Wolf (1988) found that workers had fixed lifespans regardless of time spent outside the hive, and concluded that lifespan did not depend on extrinsic mortality. In a study in which workers were trained to visit an artificial flower to collect food, and thus protected from extrinsic mortality, old foragers took longer to collect food and fly between the hive and artificial flower, again suggesting that foragers experienced a decline in flight performance due to senescence (Tolfiski 2000).

In contrast, a survival analysis of free-flying bees of known age indicated that although locomotor performance declined after about 10 days of foraging, most bees (79\%) died before reaching this age (Visscher and Dukas 1997). Moreover, mortality rate was constant with age in these bees. These observations led the authors to conclude that lifespan in these bees was limited by predation or other sources of extrinsic mortality.

In an explicit effort to compare intrinsic vs extrinsic mortality factors in the determination of worker lifespan, and using larger sample sizes than previous studies, Rueppell et al. (2007a) compared the lifespan of workers that foraged in a protected environment (flight cage) and workers that foraged in a natural setting. They also varied the amount of time bees were allowed to forage in a flight cage. Free-foraging mortality was higher than flight-cage mortality, but both groups showed increasing mortality rates with age consistent with senescence. Limiting foraging opportunities in the cage had no overall effect on lifespan. There was also a negative correlation between age at first foraging and foraging lifespan, suggestive of pre-foraging senescence. In a separate study, Rueppell et al. (2007b) assessed agedependent mortality and behavioral performance of foragers. They found that workers experienced an increase in mortality with chronological age, but their performance in behavioral assays related to foraging activity did not decline with age.
To eliminate the confounding effects of increased extrinsic hazard and energy expenditure faced by foraging bees, Remolina et al. (2007) used a social manipulation to prevent nurse bees from transitioning to foraging. They tested whether older nurses were more susceptible to different kinds of stress: starvation, heat, and oxidative damage. In this study, all forms of stress resistance decreased in older bees, and this manifestation of senescence was evident by 30 days of age. They therefore concluded that intrinsic senescence affects nurse lifespan independently of extrinsic mortality force.

Although there are a few contradictory reports, overall the evidence supports the proposition that worker bees (both nurses and foragers) exhibit senescence. However, no study has directly assessed queen-worker differences in aging rates. Corona et al. (2007) compared paraquat resistance in queens and workers of similar age and found that queens were much more resistant to this form of oxidative stress. The age chosen for this comparison (30 days) had been associated with senescence in workers (Remolina et al. 2007). These results suggest that, while workers are experiencing senescent decline in stress resistance by this age, and are not expected to live much longer, queens are highly stress resistant and expected to live more than 10-fold longer. A reasonable interpretation of this result is that at least part of the difference in queen and worker life span can be attributed to differences in intrinsic rates of aging. Nonetheless, a direct comparison of queen and worker lifespan, and assessment of stress resistance throughout the lifespan, would place the intrinsic senescence hypothesis on firmer footing.

Direct comparisons of queen and worker lifespan, and other aspects of age-related performance will be difficult, at least in honey bees. In this species, it is problematic to house queens and workers under equivalent conditions. Workers cannot be confined for prolonged periods because they must fly in order to defecate. Queens cannot be kept in isolation from workers, since they do not feed themselves. Queens are fed and groomed by nurse bees, making it difficult to house them in the laboratory for long periods. It might be possible to make rearing conditions more equivalent by, for example, restricting the amount of time workers are allowed to fly. However, energy expenditure, and other differences in lifestyle between queens and workers probably cannot be made entirely equivalent. 


\section{Molecular and physiological mechanisms of extended queen lifespan}

As outlined above, there is a well-accepted model for the evolution of long life in social insect queens. However, how queens achieve both long life and sustained high fecundity, compared to other insects, is not yet understood. Several proximate mechanisms for this phenomenon have been proposed; here we review three of the most prominent: oxidative stress defenses, immunocompetence, and endocrine signaling.

\section{Oxidative stress resistance}

The oxidative stress theory of aging (Harman 1956) states that cumulative oxidative damage causes aging and that lifespan is inversely related to the rate at which damage occurs. Therefore, a long-lived organism, such as the queen honey bee, should have a lower rate of reactive oxygen species (ROS) production, eliminate ROS more effectively, or better repair oxidative damage in order to minimize accumulation of damage. The first direct test of this hypothesis in a social insect was conducted by Parker et al. (2004), who found, contradictory to expectations, that $\mathrm{Cu}-\mathrm{Zn}$ superoxide dismutase 1 (CuZnSOD) expression and activity were lower in queen black ants (Lasius niger) compared to workers. Using honey bees, Corona et al. (2005) compared young and old queens and workers with respect to mRNA levels of eight antioxidant genes and mitochondrial proteins involved in cellular respiration in different tissues (head, thorax and abdomen). Confirming the results reported by Parker et al. (2004), antioxidant and respiratory gene expression decreased dramatically with age in queens, increased or remained unchanged with age in workers, and were generally substantially lower in old queens than in old workers. These two studies indicate that increased antioxidant expression is not a prerequisite for long lifespan in social insects, but do not rule out the possibility that increased lifespan is related to lower ROS production, an alternate mechanism of ROS scavenging (Corona et al. 2005, 2007), or better repair of oxidative damage. Another hypothesis suggested by Haddad et al. (2007) is that differences in fatty acid composition of the cell membranes of queens and workers may in part explain the differences in oxidative stress tolerance observed in the two female castes (see also Hulbert 2008, this issue).
It is possible that honey bees use less well studied molecules for ROS scavenging. Oxidative stress resistance in workers has been linked to the expression of vitellogenin ( $\mathrm{Vg}$ ) (Seehuus et al. 2006). Vitellogenin is a female-specific glycolipoprotein synthesized in the fat body of the abdomen, and transported to the ovaries and other body tissues (Amdam et al. 2003a). Seehuus et al. (2006) showed that bees with higher Vg levels survived longer after Paraquat injection, and Vg knockdown by RNAi treatment significantly shortened lifespan (Seehuus et al. 2006). Consistent with these findings, Corona et al. (2007) reported that queen honey bees, which possess higher Vg levels than any workers, also tolerate Paraquatinduced oxidative stress better than workers of comparable age.

Immunosenescence and immune system tradeoffs

Insects possess cellular and humoral immune mechanisms that identify and eliminate foreign tissue and pathogens. Cell-mediated immunity involves hemocytes, which are able to recognize and phagocytose foreign bodies present in the hemolymph. Hemocytes also have the ability to form groups that encapsulate large foreign bodies and nodules to trap invading bacteria. In addition to cell-mediated immunity, insects synthesize antibacterial peptides in the fat body in response to microbial infection (Klowden 2002). Moreover, honey bees and other social insects employ behavioral strategies, such as grooming, to fight pathogens (reviewed in Evans 2006).

A general feature of aging in animals is the deterioration of immune efficiency, or immunosenescence (Solana and Pawelec 1998). A decline with age in the encapsulation and melanization wound response has been observed in worker bumble bees with no corresponding decrease in hemocyte numbers (Doums et al. 2002). Amdam et al. (2005) reported that honey bee foragers have reduced hemocyte numbers and higher microbe and nodule numbers compared to nurses, but concluded that this difference was influenced more by behavioral role (possibly mediated by exposure to pathogens) than by age. That conclusion was based on a social manipulation that forced foragers to reverse to the nursing stage. This manipulation resulted in reverted (old) nurses that were physiologically similar to normal-age nurses (low JH titers, high $\mathrm{Vg}$ titers, enlarged hypopharyngeal glands) and had 
higher hemocyte counts than foragers of the same chronological age.

In contrast, Schmid et al. (2008) reported that hemocyte numbers decreased with age in queens, workers, and drones, and that old nurses and foragers have lower hemocyte numbers than their young counterparts, suggesting that hemocyte decline is not task- but age-dependent. Neither of these studies linked measures of immune system components directly to mortality rates or senescence, and both contrast with a report that challenges to the immune system of bumble bees, which should increase immune component expression, actually led to decreased survival under starvation conditions (Moret and Schmid-Hempel 2000). In fact, chronic immune system activation can cause decreased lifespan in the fruit fly Drosophila melanogaster (Libert 2006). Thus, it is not clear that upregulation of immune system components, should be directly associated with longer lifespan.

Endocrine signaling and the regulation of aging

The insulin insulin-like signaling (IIS) pathway regulates metabolism, development, reproduction, and longevity in model organisms, including nematode worms, fruit flies, and, and mammals (reviewed in Kenyon 2001; Partridge and Gems 2002, Hughes and Reynolds 2005; Sinclair et al. 2005). In addition to its potential effect on caste determination during development (Wheeler et al. 2006) insulin signaling has been suggested to be a key determinant of lifespan differences between honey bee queens and workers (Corona et al. 2007). Honey bees possess at least two insulin receptors (AmInR-1 and AmInR-2) and two insulin-like peptides (AmILP-1 and AmILP-2). They also have four orthologs of the nematode Caenorhabditis elegans transcription factor DAF-16 (or Drosophila FOXO) (Honeybee Genome Sequencing Consortium 2006), which has been implicated in aging and lifespan regulation. Recent studies suggest that the IIS pathway interacts with $\mathrm{Vg}$ and $\mathrm{JH}$ to regulate honey bee lifespan, and these interactions may provide the key to both the long life and high fecundity of the queen (Corona et al. 2007). Similar models of endocrine regulation have been proposed to explain life history variation between nurses and foragers and between workers in divergent selection lines (Amdam and Omholt 2003b; Amdam et al. 2007). Here we focus on endocrine signaling differences that may lead to variation in lifespan between queen and worker honey bees.

In Drosophila, JH and $\mathrm{Vg}$ abundance are positively correlated, and a calorically enriched diet causes increased signaling through the IIS pathway, which in turn shortens lifespan ( $\mathrm{Tu}$ et al. 2006). In honey bees, $\mathrm{JH}$ and $\mathrm{Vg}$ abundance are negatively correlated: low levels of $\mathrm{JH}$ in the queen allow $\mathrm{Vg}$ titers to build up, whereas high levels of $\mathrm{JH}$ in foragers causes $\mathrm{Vg}$ titers to drop (Fluri et al. 1981; Hartfelder and Engels 1998). Knockdown of Vg with RNAi caused increased circulating JH titers in workers (Guidugli et al. 2005), and application of a $\mathrm{JH}$ analog decreased $\mathrm{Vg}$ in queens (Corona et al. 2007).

The association between nutrition and lifespan is also reversed in honey bees compared to that in model organisms. In bees, enriched nutrition (in queens, and in nurses compared to foragers) is associated with low expression of insulin-like peptides and insulin receptors. This pattern suggests that, unlike in flies, worms, or mice, caloric intake in bees is negatively correlated with signaling through the IIS pathway (however, effects on IIS of direct manipulation of caloric intake have not been reported). These observations, together

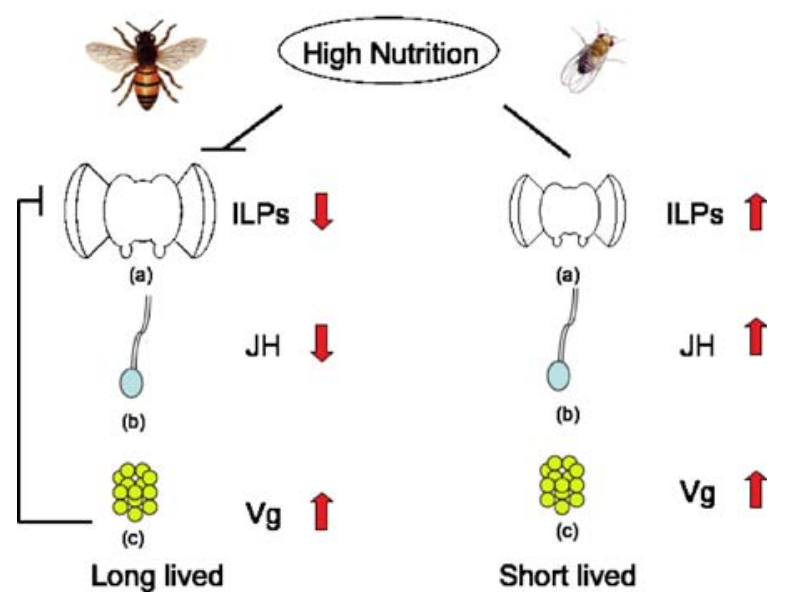

Fig. 1 Model of insulin insulin-like signaling (IIS) and lifespan regulation in adult queen honey bees compared to Drosophila. In honey bees, high nutrition leads to low signaling through the IIS and longer lifespan. Low juvenile hormone (JH) levels increase vitellogenin $(\mathrm{Vg})$ titers, which negatively regulate $\mathrm{JH}$ levels through a feedback loop which represses IIS. In fruit flies, high nutrition is associated with a decrease in lifespan. JH hormone levels are positively related to Vg levels. $a-c$ indicate brain, corpora allata, and fat bodies, respectively. Insulin-like peptides (ILPs) are produced in brain, JH in corpora allata, and $\mathrm{Vg}$ in fat bodies of insects 
with the models of endocrine regulation in workers proposed by Amdam and colleagues, led Corona et al. (2007) to propose a model for how both conserved and species-specific molecular mechanisms contribute to long life and high fecundity in queen bees. In this model, key differences between these mechanisms in honey bees and fruit flies account for the inverse relationship between nutrition and insulin signaling, the resulting low $\mathrm{JH}$ levels that induce $\mathrm{Vg}$ expression, and a proposed negative feedback loop linking high Vg with repression of the IIS pathway (Fig. 1). Under this model, low signaling through the IIS pathway accounts for queen longevity, while the ability to maintain high $\mathrm{Vg}$ titers despite low IIS signaling allows for prolonged high fecundity. While aspects of this model are untested (e.g., the idea that effects of $\mathrm{JH}$ on IIS are mediated through $\mathrm{Vg}$ ), it provides a scheme for progress in understanding proximate mechanisms underlying the unusual life history of queen honey bees.

\section{Conclusion}

Social insects in general, and honey bees in particular, provide a unique system for aging research. Based on inferences from ecological and population genetic studies, it seems likely that the striking longevity and fecundity of social insect queens evolved as a consequence of selection pressure accompanying the evolution of eusociality. Alternatively, it is possible that the causality was reversed, and that long life set the stage for the evolution of eusociality (Carey 2001); a similar hypothesis has been proposed for the evolution of cooperative breeding in birds (Arnold and Owens 1998; Ridley et al. 2005). It will probably be difficult to test these alternatives directly, although an experimental evolutionary approach might be feasible in the laboratory. For example, aspects of a eusocial lifestyle might be mimicked in a selection experiment using an originally non-eusocial organism, and evolutionary changes in lifespan could then be monitored. Admittedly, it is more difficult to imagine an equivalent experiment to test the idea that long life led to eusociality.

The physiological and molecular basis of queen longevity and the dramatic lifespan differences between queens and workers is just beginning to be explored, but shows great promise. This work has already helped to shape fundamental questions for both molecular and evolutionary biologists. For example, how have the traditional relationships between nutrition, IIS signaling, and vitellogenesis been reversed in honey bees? Does the same mechanism underlie lifespan extension in all social insects, or have different taxa evolved different mechanisms? Did novel functions of $\mathrm{Vg}$ evolve before or after the evolution of caste differentiation (Corona et al. 2007)? We look forward to the resolution of at least some of these issues in the near future.

Acknowledgments The authors wish to thank Katelyn Michelini, Scott Kreher, Deborah Kristan, Donna Holmes and one anonymous reviewer for comments that greatly improved the manuscript. The authors' work on evolutionary and mechanistic bases of aging in insects has been supported by grants from NSF, $\mathrm{NIH}$, and the University of Illinois Urbana-Champaign.

\section{References}

Anderson J (1931) How long does a bee live? Bee World $12: 25-26$

Amdam GV, Norberg KA, Hagen A, Omholt SW (2003a) Social exploitation of vitellogenin. Proc Natl Acad Sci USA 100:1799-1802

Amdam GV, Omholt SW (2003b) The hive bee to forager transition in honeybee colonies: the double repressor hypothesis. J Theor Biol 223:451-464

Amdam GV, Aase ALTO, Seehuus SC, Fondrk MK, Norberg K, Hartfelder K (2005) Social reversal of immunosenescence in honey bee workers. Exp Gerontol 40:939-947

Amdam GV, Nilsen KA, Norberg K, Fondrk MK, Hartfelder K (2007) Variation in endocrine signaling underlies variation in social life history. Am Nat 170:37-46

Arnold KE, Owens IPF (1998) Cooperative breeding in birds: a comparative test of the life history hypothesis. Proc R Soc B 265:739-745

Baudisch A (2005) Hamilton's indicators of the force of selection. Proc Natl Acad Sci USA 102:8263-8268

Bozina KD (1961) How long does the queen live? Pchelovodstvo $38: 13$

Charlesworth B (1994) Evolution in age-structured populations. Cambridge University Press. Cambridge, England

Carey JR (2001) Demographic mechanisms for the evolution of long life in social insects. Exp Gerontol 36:713-722

Corona M, Hughes KA, Weaver DB, Robinson GE (2005) Gene expression patterns associated with queen honey bee longevity. Mech Ageing Dev 126:1230-1238

Corona M, Velarde RA, Remolina S, Moran-Lauter A, Wang Y, Hughes KA, Robinson GE (2007) Vitellogenin, juvenile hormone, insulin signaling, and queen honey bee longevity. Proc Natl Acad Sci USA 104:7128-7133

Doums C, Moret Y, Benelli E, Schmid-Hempel P (2002) Senescence of immune defence in Bombus workers. Ecol Entomol 27:138-144

Evans JD, Aronstein K, Chen YP, Hetru C, Imler JL, Kanost JM, Thompson GJ, Zous Z, Hultmark D (2006) Immune 
pathways and defence mechanisms in honey bees Apis mellifera. Insect Mol Biol 15:645-656

Fluri P, Wille H, Gerig L, Lüscher M (1977) Juvenile hormone, vitellogenin and haemocyte count in winter worker honeybees (Apis mellifera). Cell Mol Life Sci 33:1240-141

Fluri P, Sabatini AG, Vecchi MA, Wille H (1981) Blood juvenile hormone, protein and vitellogenin titres in laying and non-laying queen honeybees. J Apic Res 20:221-225

Free JB, Spencer-Booth Y (1959) The longevity of worker honey bees (Apis mellifera). Proc R Entomol Soc 34:141-150

Fukuda H, Sekiguchi K (1966) Seasonal change of the honeybee worker longevity in Sapporo, north Japan, with notes on some factors affecting lifespan. Jpn J Ecol 16:206-212

Guidugli KR, Nascimento AM, Amdam GV, Barchuk AR, Omholt S, Simoes ZLP, Hartfelder K (2005) Vitellogenin regulates hormonal dynamics in the worker caste of a eusocial insect. FEBS Lett 579:4961-4965

Haddad LS, Kelbert L, Hulbert LJ (2007) Extended longevity of queen honey bees compared to workers is associated with peroxidation-resistant membranes. Exp Gerontol 42:601-609

Harman D (1956) Aging: a theory based on free radical and radiation chemistry. J Gerontol 11:298-300

Hartfelder K, Engels W (1998) Social insect polymorphism: hormonal regulation of plasticity in development and reproduction in the honeybee. Current Top Dev Biol 40:45-77

Hölldobler B, Wilson EO (1990) The Ants. Harvard University Press. Cambridge, Massachusetts

Huang ZY, Robinson GE (1996) Regulation of honey bee division of labor by colony age demography. Behav Ecol Sociobiol 39:147-158

Hughes KA, Reynolds RM (2005) Evolutionary and mechanistic theories of aging. Annu Rev Entomol 50:421-445

Hulbert AJ (2008) Explaining longevity of different animals: is membrane fatty acid composition the missing link? AGE (in press) DOI 10.1007/s11357-008-9055-2

Keller L, Jemielity S (2006) Social insects as a model to study the molecular basis of ageing. Exp Gerontol 41:553-556

Keller L, Genoud M (1997) Extraordinary lifespans in ants: a test of evolutionary theories of ageing. Nature 389:958-960

Keller L (1998) Queen lifespan and colony characteristics in ants and termites. Insectes Soc 45:235-246

Kenyon C (2001) A conserved regulatory system for aging. Cell 105:165-168

Klowden MJ (2002) Physiological systems in insects. Elsevier, San Diego

Libert S, Chao Y, Chu X, Pletcher SD (2006) Trade-offs between longevity and pathogen resistance in Drosophila melanogaster are mediated by NFKB signaling. Aging Cell 5:533-546

Merrill JH (1924) Observations on brood-rearing. Am Bee J 64:337-338

Moret Y, Schmid-Hempel P (2000) Survival for immunity: the price of immune system activation for bumblebee workers. Science 290:1166-1168

Neukirch A (1982) Dependence of the lifespan of the Honeybee (Apis mellifica) upon flight performance and energy consumption. J Comp Physiol 146:35-40

Nolan WJ (1925) The brood-rearing cycle of the honey bee. Bull US Dept Agric 1349:1-56
Page RE, Peng CYS (2001) Aging and development in social insects with emphasis on the honey bee, Apis mellifera $\mathrm{L}$. Exp Gerontol 36:695-711

Parker JD, Parker KM, Sohal BH, Sohal RS, Keller L (2004) Decreased expression of $\mathrm{Cu}-\mathrm{Zn}$ superoxide dismutase 1 in ants with extreme life-span. Proc Natl Acad Sci USA 101:3486-3489

Partridge L, Gems D (2002) Mechanisms of aging: public or private? Nat Rev Genet 3:165-175

Patel A, Fondrk MK, Kaftanoglu O, Emore C, Hunt G, Frederick K, Amdam GV (2007) The making of a queen: TOR pathway is a key player in diphenic caste development. PLoS ONE 2:e509

Remolina SC, Hafez DM, Robinson GE, Hughes KA (2007) Senescence in the worker honey bee Apis mellifera. J Insect Physiol 53:1027-1033

Ribbands CR (1952) The division of labour in the honeybee community. Proc R Soc Lond B 140:32-43

Ridley J, Yu DW, Sutherland WJ (2005) Why long-lived species are more likely to be social: the role of local dominance. Behav Ecol 16:358-363

Robinson GE, Page RE, Strambi C, Strambi A (1989) Hormonal and genetic control of behavioral integration in honeybee colonies. Science 246:109-112

Robinson GE, Strambi C, Strambi A, Feldlaufer MF (1991) Comparison of juvenile hormone and ecdysteroid adult haemolymph titres in adult worker and queen honey bees (Apis mellifera). J Insect Physiol 37:929-935

Robinson GE (1992) Regulation of division of labor in insect societies. Annu Rev Entomol 37:637-665

Roff DA (2002) Life history evolution. Sinauer, Massachusetts

Rueppell O, Bachelier C, Fondrk MK, Page RE Jr (2007a) Regulation of life history determines lifespan of workers honey bees (Apis mellifera L.). Exp Gerontol 42: 1020-1032

Rueppell O, Christine S, Mulcrone C, Groves L (2007b) Aging without functional senescence in honey bee workers. Curr Biol 17:R274-R275

Rutz W, Lüscher M (1974) The occurrence of vitellogenin in workers and queens of Apis mellifica and the possibility of its transmission to the queen. J Insect Physiol 20:897-909

Schmid MR, Brockmann A, Pirk CWW, Stanley DW, Tautz J (2008) Adult honey bees (Apis mellifera L.) abandon hemocytic, but not phenoloxidase-based immunity. J Insect Physiol 54:439-444

Schmid-Hempel P, Wolf T (1988) Foraging effort and lifespan of workers in a social insect. J Anim Ecol 57:509-521

Seehuus SC, Norberg K, Gimsa U, Krekling T, Amdam GV (2006) Reproductive protein protects functionally sterile honey bee workers from oxidative stress. Proc Natl Acad Sci USA 103:962-967

Sinclair DA (2005) Toward a unified theory of caloric restriction and longevity regulation. Mech Ageing Dev 126:987-1002

Solana R, Pawelec G (1998) Molecular and cellular basis of immunosenescence. Mech Ageing Dev 102:115-129

Stearns SC (1992) The evolution of life histories. Oxford University Press. New York

The Honeybee Genome sequencing Consortium (2006) Insights into social insects from the genome of the honeybee Apis mellifera. Nature 443:931-949 
Tolfiski A (2000) Senescence and learning in honeybee (Apis mellifera) workers. Acta Neurobiol Exp 60:35-39

Toth AL, Robinson GE (2005) Worker nutrition and division of labour in honeybees. Anim Behav 69:427-435

Tu MP, Flatt T, Tatar M (2006) Juvenile and steroid hormones in Drosophila melanogaster longevity. In: Masoro EJ, Austad SN (eds) Handbook of the biology of aging, 6th edn. Academic, San Diego

Visscher PK, Dukas R (1997) Survivorship of foraging honeybees. Insect Soc 44:1-5
Wheeler DE, Buck N, Evans J (2006) Expression of insulin pathway genes during the period of caste determination in the honey bee, Apis mellifera. Insect Mol Biol 15: 597-602

Wilson EO (1971) The insect societies. Belknap Press of Harvard University Press. Cambridge, Massachusetts

Winston ML (1987) The biology of the honey bee. Harvard University Press. Cambridge, MA

Woyke J (1960) Natural and artificial insemination of queen honey bees. Pszczelnicze Zeszyty Nauwoke 4:183-273 